\title{
MEASURES OF CARLESON TYPE ON FILTRATED PROBABILITY SPACES AND THE CORONA THEOREM ON COMPLEX BROWNIAN SPACES
}

\author{
HITOSHI ARAI
}

\begin{abstract}
N. Th. Varopoulos showed the existence of corona solutions for bounded holomorphic martingales (cf. [7]). In this paper we investigate measures of Carleson type on usual filtrated probability spaces and as its application we estimate the corona solutions by a probabilistic modification of Wolff's approach.
\end{abstract}

1. Introduction. Let $(\Omega, \mathcal{F}, P)$ be a complete probability space with a nondecreasing right continuous family $(\mathcal{F}(t) ; t \geq 0)$ of sub- $\sigma$-fields of $\mathcal{F}$ such that $\mathcal{F}(0)$ contains all $P$-null sets. Assume that $\mathcal{F}=\mathcal{F}(\infty)\left(\equiv \bigvee_{t>0} \mathcal{F}(t)\right)$.

Let $\mu$ be a complex measure on $\bar{R}_{+} \times \Omega$, where $\bar{R}_{+}=[0, \infty)$. We call the measure $\mu$ Carleson type if $\|\mu\|_{c} \equiv \sup \{|\mu|(S(T)) / P(T<\infty): T$ is an $(\mathcal{F}(t))$-stopping time with $P(T<\infty) \neq 0\}<\infty$, where $S(T)=\{(t, \omega): T(\omega) \leq t<\infty\}$, and where $|\mu|$ is the total variation of $\mu$.

In this paper we study measures of the above type and give an application to the corona theorem on complex Brownian spaces. In $\S 2$ we give a characterization of measures of Carleson type. In $\S 3$, by using results of $\S 2$ we prove our main theorem, Theorem 3, which is a probabilistic modification of Wolff's theorem relating to the corona theorem for bounded holomorphic functions on the unit disc. From our probabilistic modification we give in $\S 3$ an estimate of corona solutions obtained by N. Th. Varopoulos [7]. Our main theorem is different from a direct probabilistic approach of the theorem of Wolff. If one tries to estimate the corona solutions by a probabilistic version of Wolff, one needs to modify this approach, since even in the case of Brownian filtrated probability spaces we cannot use the normal family argument and the integral operator $T(G)(z)=(2 \pi i) \iint_{|\zeta|<1} G(\varsigma)(\zeta-z)^{-1} d \zeta \wedge d \bar{\zeta}$.

2. Measures of Carleson type. Here we use notation as in $\S 1$. Let $\mathcal{M}^{p}=$ $\left\{X=\left(X_{t}\right)_{t \geq 0}: X\right.$ is an $\left(\xi_{t}\right)$-martingale with $\left.X^{*} \equiv \sup _{t}\left|X_{t}\right| \in L^{p}(\Omega)\right\}, 0<p<\infty$.

The following theorem is a probabilistic analogue of Carleson's theorem and characterizes measures of Carleson type.

THEOREM 1. Let $\mu$ be a complex measure on $\bar{R}_{+} \times \Omega$. Then the following are equivalent.

(1) $\mu$ is a measure of Carleson type.

Received by the editors January 14, 1985.

1980 Mathematics Subject Classification. Primary 46J15, 60G45.

Key words and phrases. Measure of Carleson type, BMO-martingale, holomorphic martingale, Wolff's theorem, corona theorem. 
(2) For every $p \in(0, \infty)$ and for every $X \in \mathcal{M}^{p}$,

$$
\iint|X|^{p}|\mu|(d t, d \omega) \leq C|| X^{*} \|_{p}^{p}
$$

where $C$ is a constant depending only on $\mu$.

(3) For some $p \in(1, \infty)$ and for every $X \in \mathcal{M}^{p}$,

$$
\iint|X|^{p}|\mu|(d t, d \omega) \leq C_{p}\left\|X_{\infty}\right\|_{p}^{p}
$$

where $C_{p}$ is a constant depending only on $\mu$ and $p$.

Here we can take $\|\mu\|_{c}$ and $(p /(p-1))^{p}\|\mu\|_{c}$ as $C$ and $C_{p}$, respectively.

PROOF. (1) $\rightarrow$ (2) Let $\lambda>0$ be an arbitrary positive number. Let $T=$ $\inf \left\{t:\left|X_{t}\right|>\lambda\right\}$. Since $\left\{\left|X_{s}\right|>\lambda\right\} \subset\{T \leq s\}$, we have $\left\{(s, \omega):\left|X_{s}(\omega)\right|>\lambda\right\} \subset$ $S(T)$. Hence

$$
|\mu|\left(\left\{(s, \omega):\left|X_{s}(\omega)\right|>\lambda\right\}\right) \leq|\mu|(S(T)) \leq\|\mu\|_{c} P(T<\infty) \leq\|\mu\|_{c} P\left(X^{*}>\lambda\right) .
$$

Thus we obtain $\iint|X|^{p}|\mu|(d t, d \omega) \leq\|\mu\|_{c}\left\|X^{*}\right\|_{p}^{p}$.

$(2) \rightarrow(3)$ This is clear by Doob's inequality.

$(3) \rightarrow(1)$ Let $T$ be an arbitrary $\left(\mathcal{F}_{t}\right)$-stopping time. Let $X_{t}=E\left[\chi_{\{T<\infty\}} \mid \mathcal{F}_{t}\right](t \geq$ 0 ), where $\chi$. is the characteristic function of .. Then $X_{t} \geq E\left[\chi_{\{T \leq t\}} \mid \mathcal{F}_{t}\right]=\chi_{\{T \leq t\}}$, $t \geq 0$.

Hence

$$
\begin{aligned}
|\mu|(S(T)) & =\iint \chi_{\{T \leq t\}}|\mu|(d t, d \omega) \leq \iint\left|X_{t}\right|^{p}|\mu|(d t, d \omega) \\
& \leq C_{p}\left\|X_{\infty}\right\|_{p}^{p} \leq C_{p} P(T<\infty) .
\end{aligned}
$$

Now we study a relation between measures of Carleson type and continuous BMO-martingales. If $X$ is a continuous $L^{2}$ bounded martingale, then we set $\mu_{X}(L)=E\left[\int_{0}^{\infty} \chi_{L} d\langle X, X\rangle\right], L \subset R_{+} \times \Omega$. The following theorem is an immediate consequence of the observation in $[4$, p. 333].

THEOREM 2. For every continuous $L^{2}$ bounded martingale $X, X$ is BMO if and only if $\mu_{X}$ is Carleson type. Then we have $\left\|X-X_{0}\right\|_{\mathrm{BMO}}^{2}=\left\|\mu_{X}\right\|_{c}$, where $\|X\|_{\mathrm{BMO}}=\sup _{t} \| E\left[\left|X-X_{t}\right|^{2} \mid \mathcal{F}_{t}\right]||_{\infty}^{1 / 2}+\left|X_{0}\right|$.

The relation between measures of Carleson type and Carleson measures on domains of $\mathbf{R}^{n}$ is deep. We will study the relation and give applications to the harmonic analysis in a forthcoming paper.

3. Probabilistic modification of Wolff's theorem. Let $\left(z_{1}(t): t \geq 0\right), \ldots$, $\left(z_{m}(t): t \geq 0\right)$ be $m$ independent complex Brownian motions on a complete probability space $(\Omega, P)$ such that $P\left(z_{j}(0)=0 ; j=1, \ldots, m\right)=1$. For every $t \geq 0, \mathcal{F}(t)$ denotes the $\sigma$-field generated by $\left\{z_{j}(s): 0 \leq s \leq t ; j=1, \ldots, m\right\}$ and all $P$-null sets, and $\mathcal{F}$ denotes the $\sigma$-field generated by $\bigcup_{t>0} \mathcal{F}(t)$.

Let $\partial / \partial z_{j}=\left(\left(\partial / \partial x_{j}\right)-(-1)^{1 / 2}\left(\partial / \partial y_{j}\right)\right) / 2$, where $x_{j}=\operatorname{Re} z_{j}$ and $y_{j}=\operatorname{Im} z_{j}$, $j=1, \ldots, m$. For an Ito process $G$ (cf. $[\mathbf{3}])$, let $\bar{D} G=\sum_{j=1}^{m}\left(\partial G / \partial \bar{z}_{j}\right) d \bar{z}_{j}+$ $(\partial G / \partial t) d t$ (the bar denotes conjugation, here and always). 
The following spaces were introduced by Varopoulos [7]:

$$
H^{p} \equiv\left\{X_{\infty} \in L^{p}(\Omega): X=\left(X_{t}=E\left[X_{\infty} \mid F_{t}\right]\right) \text { satisfies } \bar{D} X=0\right\}, \quad 1 \leq p \leq \infty .
$$

Our main theorem is the following which is a probabilistic modification of a theorem of Wolff [2, VIII, Theorem 1.2].

THEOREM 3. Suppose $1 \leq p<\infty$. Let $G$ be an Ito process (resp. a martingale) satisfying

(1) $E\left[\left(\int_{0}^{\infty} \sum_{j=1}^{m}\left|\left(\partial G / \partial z_{j}\right)\right|^{2} d t\right)^{p / 2}\right]<\infty$,

(2) two measures $d \mu=\sum_{j=1}^{m}\left|\left(\partial G / \partial \bar{z}_{j}\right)\right|^{2} d t d P$ and $d \nu=|(\partial G / \partial t)| d t d P$ are Carleson type.

Then there exists an $a_{\infty} \in H^{1}$ (resp. $\left.a_{\infty} \in H^{p}\right)$ with

$$
\left\|a_{\infty}+G_{\infty}\right\|_{\infty} \leq C_{1}\left(\|\mu\|_{c}\right)^{1 / 2}+C_{2}\|\nu\|_{c}
$$

where $C_{1}$ and $C_{2}$ are absolute constants.

For the proof of Theorem 3 we need the following lemmas.

LEMMA 1. If $X_{\infty} \in H^{1}$ with $X_{0} \neq 0$, then there exist $q_{\infty} \in H^{\infty}$ and $h_{\infty} \in H^{2}$ satisfying $\left|q_{\infty}\right|=1$ a.s., $E\left[\log \left(\left|h_{\infty}\right|\right)\right]=\log \left(\left|h_{0}\right|\right)>-\infty$ and $X_{t}=q_{t}\left(h_{t}\right)^{2}, 0 \leq t \leq$ $\infty$. 1].

This lemma is an immediate consequence of the factorization theorem in $[6$ or

The following lemma is proved by using $[\mathbf{7}$, Theorem $3.1 ; \mathbf{6}$, Theorem 3.1 , Corollary 2.4.6; 5 , Theorem 1 ]. But we omit the proof.

LEMMA 2. Let $\Lambda_{0} \equiv\left\{\Phi: \Phi\right.$ is a simple process, that is, $\Phi=\sum_{j=1}^{n} \phi_{j} \chi_{(\sigma(j), \tau(j)]}$, where $\sigma(j)$ and $\tau(j)$ are positive numbers with $\sigma(j)<\tau(j)$ and $\phi_{j} \in L^{\infty}\left(\Omega, \mathcal{F}_{\sigma(j)}, P\right)$ $(j=1, \ldots, n)$ where $n$ is a positive integer $\}$.

For $a \Phi \in \Lambda_{0}$, we set $T(\Phi, l, j)=\inf \left\{t:\left|\int_{0}^{t} \Phi d z_{j}\right|>l\right\}(l>0)$. Let $S^{\infty} \equiv$ linear $\operatorname{span}\left\{X_{\infty}: X_{t}=c+\sum_{j=1}^{m} \int_{0}^{T(\Phi(j), l(j), j) \wedge t} \Phi(j) d z_{j}\right.$ for some $\Phi(j) \in \Lambda_{0}, c \in \mathbf{C}$ and $l(j)>0 ; j=1, \ldots, m\}$, where $\mathbf{C}$ is the complex number field. Then

(1) $S^{\infty}$ is a subspace of $H^{\infty}$.

(2) For every $1 \leq p<\infty, S^{\infty}$ is $L^{p}$-norm dense in $H^{p}$.

(3) The algebra generated by $S^{\infty}$ is a weak $k^{*}$ Dirichlet algebra on $(\Omega, \mp, P)$ and weak $k^{*}$ dense in $H^{\infty}$. (See [6] for the definition of weak* Dirichlet algebras.)

Let us denote by $\nvdash^{\infty}$ the algebra generated by $S^{\infty}$. The element $X_{\infty}$ of $\nvdash^{\infty}$ has a nice property, that is, two functions $\sup _{t}\left|X_{t}\right|$ and $\sup _{t}\left|\left(\partial X / \partial z_{j}\right)_{t}\right|$ are essentially bounded.

Now we are ready to prove the main result of this paper, Theorem 3. This is obtained by combining ideas of Wolff (cf. [2, p. 322]) and Varopoulos (cf. [7, p. 117]). For the proof we use essentially Theorems 1 and 2.

To simplify notation we will suppose that $m=1$, however, the proof holds in general. We denote $z=z_{1}$.

Let $F_{t}=\int_{0}^{t}(\partial G / \partial \bar{z}) d \bar{z}+\int_{0}^{t}(\partial G / \partial t) d t, t \geq 0$. Take any $K_{\infty} \in H^{\infty}$ with $\left\|K_{\infty}\right\|_{1} \leq 1$. There exists a positive number $\varepsilon<1$ with $K_{0}+\varepsilon \neq 0$. Let $L=K+\varepsilon$. By Lemma 1 we obtain two functions $q_{\infty} \in H^{\infty}$ and $h_{\infty} \in H^{\infty}$ with $\left|q_{\infty}\right|=1$ a.s. and $L_{t}=q_{t}\left(h_{t}\right)^{2}, 0 \leq t \leq \infty$. Then

$$
(\partial q / \partial z)\left(h^{2}\right)+2 q h(\partial h / \partial z)=(\partial L / \partial z)=(\partial K / \partial z) .
$$


By Theorems 1 and 2 we get

$$
\begin{gathered}
E\left[\int_{0}^{\infty}|h|^{2}|(\partial F / \partial \bar{z})|^{2} d t\right] \leq 4\|\mu\|_{c}\left\|h_{\infty}\right\|_{2}^{2}=4\|\mu\|_{c}\left\|L_{\infty}\right\|_{1} \leq 8\|\mu\|_{c} \\
E\left[\int_{0}^{\infty}|h|^{2}|(\partial q / \partial z)|^{2} d t\right] \leq 4\|q\|_{\mathrm{BMO}}^{2}\left\|h_{\infty}\right\|_{2}^{2} \neq C_{3}\left\|q_{\infty}\right\|_{\infty}^{2}\left\|K_{\infty}\right\|_{1} \leq C_{3},
\end{gathered}
$$

where $C_{3}$ is an absolute constant,

$$
\begin{gathered}
E\left[\int_{0}^{\infty}|q h|^{2}|\partial F / \partial \bar{z}|^{2} d t\right] \leq 4\|\mu\|_{c}\left\|q_{\infty} h_{\infty}\right\|_{2}^{2} \leq 4\|\mu\|_{c}\left\|h_{\infty}\right\|_{2}^{2} \leq 8\|\mu\|_{c} \\
E\left[\int_{0}^{\infty}|\partial h / \partial z|^{2} d t\right] \leq 20\left\|K_{\infty}\right\|_{1} \leq 20, \quad \text { and } \\
E\left[\int_{0}^{\infty}|K||\partial F / \partial t| d t\right] \leq\|\nu\|_{c}\left\|K^{*}\right\|_{1} \leq C_{4}\|\nu\|_{c}\left\|K_{\infty}\right\|_{1} \leq C_{4}\|\nu\|_{c}
\end{gathered}
$$

where $C_{4}$ is an absolute constant. Thus

$$
\begin{aligned}
&\left|E\left[K_{\infty} F_{\infty}\right]\right| \leq\left|E\left[\int_{0}^{\infty} K(\partial F / \partial t) d t\right]\right|+2\left|E\left[\int_{0}^{\infty}(\partial K / \partial z)(\partial F / \partial \bar{z}) d t\right]\right| \\
& \leq C_{4}\|\nu\|_{c}+2\left|E\left[\int_{0}^{\infty}(\partial q / \partial z)\left(h^{2}\right)(\partial F / \partial \bar{z}) d t\right]\right| \\
&+4\left|E\left[\int_{0}^{\infty} q h(\partial h / \partial z)(\partial F / \partial \bar{z}) d t\right]\right| \\
& \leq C_{4}\|\nu\|_{c}+2\left(E\left[\int_{0}^{\infty}|h|^{2}|\partial q / \partial z|^{2} d t\right]\right)^{1 / 2}\left(E\left[\int_{0}^{\infty}|h|^{2}|\partial F / \partial \bar{z}|^{2} d t\right]\right)^{1 / 2} \\
&+4\left(E\left[\int_{0}^{\infty}|\partial h / \partial z|^{2} d t\right]\right)^{1 / 2}\left(E\left[\int_{0}^{\infty}|q h|^{2}|\partial F / \partial \bar{z}|^{2} d t\right]\right)^{1 / 2} \\
& \leq C_{4}\|\nu\|_{c}+2\left(8 C_{3}\|\mu\|_{c}\right)^{1 / 2}+4\left(160\|\mu\|_{c}\right)^{1 / 2} \\
&= C_{1}\left(\|\mu\|_{c}\right)^{1 / 2}+C_{2}\|\nu\|_{c} \quad(\equiv A) .
\end{aligned}
$$

Let $\phi\left(f_{\infty}\right)=E\left[f_{\infty} F_{\infty}\right]$ for every $f_{\infty} \in \nvdash^{\infty}$. By the above observations, $\phi$ is a linear functional on $\mathfrak{H}^{\infty}$ with $\sup \left\{\left|\phi\left(f_{\infty}\right)\right|: f_{\infty} \in \mathfrak{H}^{\infty},\left\|f_{\infty}\right\|_{1} \leq 1\right\} \leq A$.

By the Hahn-Banach theorem there exists a $u_{\infty} \in L^{\infty}$ such that

$$
E\left[f_{\infty}\left(F_{\infty}-K^{a}(u)_{\infty}^{-}\right)\right]=0
$$

for every $f_{\infty} \in \not^{\infty}$ and $\left\|u_{\infty}\right\|_{\infty} \leq A$, where $K^{a}=\left(I+(-1)^{1 / 2} H\right) / 2$; here $H$ is the Hilbert transform defined in [7]. Hence from Lemma 2(3) and [6, Theorem 2.3.8] it follows that $F_{\infty}-\left(u_{\infty}-K^{a}(u)_{\infty}\right)=F_{\infty}-K^{a}(u)_{\infty}^{-} \in H^{1}$.

Let $a_{\infty}=-F_{\infty}+u_{\infty}-\int_{0}^{\infty}(\partial G / \partial z) d z-G_{0}$. Then $a_{\infty} \in H^{1}$ and $a_{\infty}+G_{\infty}=u_{\infty}$. If $G$ is a martingale, then it is easy to see that the function $a_{\infty}$ is in $H^{p}$.

COROLlaRY 1. Let $G$ be an Ito process and let $d \mu=|G| d t d P$ be a measure of Carleson type. Then there exists an Ito process $b$ satisfying $\bar{D} b=(\partial b / \partial t) d t=|G| d t$ and $\left\|b_{\infty}\right\|_{\infty} \leq C\|\mu\|_{c}$, where $C$ is an absolute constant.

This corollary is a probabilistic analogue of [2, VIII, Theorem 1.1]. We omit the proof. We can also prove another variant of Corollary 1. 
4. An estimate of Varopoulos's corona solutions. In this section we prove

THEOREM 4. Let $\delta \in(0,1)$ and suppose $f_{1}, \ldots, f_{n} \in H^{\infty}$ satisfy

$$
\max _{1 \leq j \leq n}\left\|f_{j}\right\|_{\infty} \leq 1 \text { and } \max _{1 \leq j \leq n} \inf _{t \geq 0}\left|E\left[f_{j} \mid \mathcal{F}_{t}\right]\right| \geq \delta \quad \text { a.s. }
$$

Then there exist $g_{1}, \ldots, g_{n} \in H^{\infty}$ satisfying

(1) $\sum_{j=1}^{m} f_{j} g_{j}=1$ a.s. (Varopoulos [7]).

(2) $\max _{1 \leq j \leq n}\left\|g_{j}\right\|_{\infty} \leq C\left(n^{3 / 2} \delta^{-3}+n^{2} \delta^{-4}\right)$, where $C$ is an absolute constant.

Here we prove the theorem only for $m=1$. For any $m$ we can prove the theorem in a simple modification of the proof of the case $m=1$.

Let $z=z_{1}$. For an $f \in H^{p}$ we denote by $f^{\prime}$ the stochastic derivative $\partial f / \partial z$ of $f$. The following lemma was shown by N. Th. Varopoulos.

LEMMA (VAROPOULOS [7]). Let $|F|^{2}=\left(\sum_{l=1}^{n}\left|f_{l}\right|^{2}\right)$ and let

$$
\begin{gathered}
\mu_{i j}=\left(f_{i} f_{j}^{\prime}-f_{j}^{\prime} f_{i}\right)^{-} /|F|^{4}, \quad \nu_{i j}=4 \sum_{l=1}^{n} \bar{f}_{l} f_{l}^{\prime}\left(f_{j} f_{i}^{\prime}-f_{i} f_{j}^{\prime}\right)^{-} /|F|^{6}, \\
u_{i j}=\int \mu_{i j} d \bar{z}+\int \nu_{i j} d t, \quad l_{i}=\left(\bar{f}_{i} /|F|^{2}\right)+\sum_{j=1}^{n} u_{i j} f_{j} \quad(i, j=1, \ldots, n) .
\end{gathered}
$$

Then $l_{i} \in H^{2}$ and $\sum_{j=1}^{n} f_{j} l_{j}=1$ a.s.

PROOF OF THEOREM 4. It is clear that

$$
\left|\mu_{i j}\right|^{2} \leq \delta^{-6}\left(\sum_{l=1}^{n}\left|f_{l}^{\prime}\right|^{2}\right) \quad \text { and }\left|\nu_{i j}\right| \leq \delta^{-4}\left(\sum_{l=1}^{n}\left|f_{l}^{\prime}\right|^{2}\right)
$$

By Theorem 2 we have $\left\|\sum_{l=1}^{n}\left|f_{l}^{\prime}\right|^{2} d t d P\right\|_{c} \leq \sum_{l=1}^{n}\left\|f_{l}-f_{l, 0}\right\|_{\text {BMO }}^{2} \leq C_{1} n$, where $C_{1}$ is an absolute constant. Fix $i<j$. By Theorem 3, there exists an $a_{i j} \in H^{1}$ such that $\left\|a_{i j, \infty}+u_{i j, \infty}\right\|_{\infty} \leq C_{1} n^{1 / 2} \delta^{-3}+C_{2} n \delta^{-4}$.

If $i>j$, then we set $a_{i j}=-a_{j i}$. Let $a_{i i}=0$ and $g_{i}=l_{i}+\sum_{j=1}^{n} a_{i j} f_{j}$. Then $\sum_{j=1}^{n} g_{j} f_{j}=1$ a.s. and $g_{j} \in H^{\infty}$ with $\left\|g_{j}\right\|_{\infty} \leq\left(1+C_{1} n^{3 / 2} \delta^{-3}+C_{2} n^{2} \delta^{-4}\right)$.

ACKNOWLEDGEMENT. I would like to express my deepest gratitude to Professor Junzo Wada for his valuable advice and encouragement.

\section{REFERENCES}

1. H. Arai, On the algebra of bounded holomorphic martingales, Proc. Amer. Math. Soc. (to appear).

2. J. B. Garnett, Bounded analytic functions, Academic Press, New York, 1981.

3. N. Ikeda and S. Watanabe, Stochastic differential equations and diffusion processes, North-Holland, Amsterdam, 1981.

4. P. A. Meyer, Un cours sur les intégrales stochastiques, Lecture Notes in Math., Vol. 511, Springer-Verlag, Berlin and New York, 1976, pp. 245-400.

5. T. Nakazi, Hardy spaces and Jensen measures, Trans. Amer. Math. Soc. 274 (1982), 375378.

6. T. P. Srinivasan and Ju-Kwei Wang, Weak* Dirichlet algebras, Proc. Internat. Sympos. on Function Algebras, Scott-Foresman, Glenview, Ill., 1966, pp. 216-249.

7. N. Th. Varopoulos, The Helson-Szegö theorem and $A_{p}$-functions for Brownian motion and several variables, J. Funct. Anal. 39 (1980), 85-121.

DePaRTMENT OF MATHEMATICS, SCHOOL OF EdUCATION, WASEda UNiVERSiTy, 1-6-1 NISHI-WASEDA, SHINJUKU-KU, TOKYO 160, JAPAN 